Voix et Images

volxetimages

\title{
Trois générations de femmes : le reflet mère-fille dans quelques nouvelles de Gabrielle Roy
}

\section{Paula Gilbert Lewis}

Volume 10, numéro 3, printemps 1985

André Major

URI : https://id.erudit.org/iderudit/200522ar

DOI : https://doi.org/10.7202/200522ar

Aller au sommaire du numéro

Éditeur(s)

Université du Québec à Montréal

ISSN

0318-9201 (imprimé)

1705-933X (numérique)

Découvrir la revue

Citer cet article

Gilbert Lewis, P. (1985). Trois générations de femmes : le reflet mère-fille dans quelques nouvelles de Gabrielle Roy. Voix et Images, 10(3), 165-176.

https://doi.org/10.7202/200522ar d'utilisation que vous pouvez consulter en ligne.

https://apropos.erudit.org/fr/usagers/politique-dutilisation/ 


\title{
Trois générations de femmes: le reflet mère-fille dans quelques nouvelles de Gabrielle Roy
}

\author{
par Paula Gilbert Lewis, Howard University
}

Dans une étude bien connue et très lue, intitulée The Reproduction of Mothering, Nancy Chodorow défend la thèse selon laquelle le maternage consiste en un don de soi envers sa progéniture. Selon Chodorow, la capacité des femmes à assumer un tel rôle provient d'attitudes intériorisées puisque ces femmes ont été formées en conséquence par leur mère. Cependant, ce processus d'intériorisation entraîne bien souvent des déformations, des interdits, des transformations et, en outre, peut être exprimé par l'intermédiaire du fantasme et du conflit. De telles réactions sont particulièrement complexes quand une femme donne naissance à une fille parce que, selon Chodorow, les mères voient en leurs filles le prolongement d'ellesmêmes. Il s'agit ici d'un rapport semi-symbiotique de nature plus narcissique que celui qui existerait entre une mère et son fils1.

Quant aux filles, elles se perçoivent comme étant plus dépendantes de leur mère et, par conséquent, moins autonomes que le sont les fils par rapport à leur mère. Voilà pourquoi, d'après Chodorow, le champ du moi chez les jeunes filles est plus perméable, plus malléable et même plus vague que celui des garçons. Elles s'identifient davantage aux autres que ces derniers. Sans jamais entièrement refouler ou abandonner leur attachement oedipien envers leur mère, les filles grandissent et luttent pour acquérir leur autonomie. Cependant, elles se soucient des rapports avec les autres et intériorisent les besoins et les sentiments de ceux-ci. Si l'identification masculine est dominée par la culture et l'affirmation de l'individualité, l'identification féminine, elle, est dominée par le rapport avec la mère 2 .

Dans un essai récent intitulé In a Different Voice, Carol Gilligan définit, à l'instar de Chodorow, la masculinité en tant qu'autonomie menacée d'intimité et la féminité en tant qu'attachement menacé d'autonomie. Les femmes assument, ainsi, leurs droits et leurs responsabilités sous l'angle de «la logique psychologique des rapports ${ }^{3}$ ».

Dorothy Dinnerstein décrit le rapport mère-fille dans la même perspective: l'affirmation de tout «Je» s'articule par rapport à un «Autrui» (non-identifié et non sexué: $I t$ ), dont la mère est la première représentante. Une fois qu'une fille s'est identifiée à cet «Autrui», elle ne cesse de se voir et de voir les autres filles comme des êtres possédant un «Je» incomplet. La mère-«Autrui» est, en outre, le premier "Tu» qui non seulement affirme, définit et limite la conscience $d u$ «Je» de la fille, mais aussi se reflète dans celui-ci. Toute tentative de sa part en vue d'acquérir sa propre autonomie de femme est par conséquent, une lutte à la fois contre le parent du même sexe et contre elle-même 4 . On met souvent en scène cette hostilité des filles à l'égard de leur mère dans la littérature du $\mathrm{XX}^{\mathrm{e}}$ siècle car, selon les propos d'Adrienne Rich, «cette perte que subit la fille dans ses rapports avec sa 
mère et celle que subit la mère dans ses rapports avec la fille représentent la tragédie essentielle de la femme ${ }^{5}$ \%.

Dinnerstein et Rich reconnaissent, néanmoins, la possibilité d'une solidarité entre femmes: les filles peuvent s'identifier à leur mère ou aux femmes en général. La solidarité entre mère et fille précède la solidarité entre femmes. Une connaissance subconsciente, préverbale, subversive s'échangeait déjà entre deux corps identiques, entre la multiplicité et l'interdépendance du «Toi. Moi.» de Luce Irigaray6. Si le conflit mère-fille demeure l'objet d'un examen rigoureux de la part des femmes dans leurs écrits actuels, le lien mère-fille - incluant souvent le lien entre femmes est également analysé dans d'autres ouvres.

Dans les deux cas, la femme est confrontée à sa propre image et à celle d'une autre femme, dont les reflets peuvent être refoulés, détournés, volatilisés, intériorisés ou acceptés par elle. Dans une étude intéressante qui traite du concept du reflet mère-fille dans deux romans antillais écrits par des femmes - Simone Schwarz-Bart, noire, francophone de la Guadeloupe et Jean Rhys, blanche, anglophone des Iles du Vent - Ronnie Scharfman montre que la structure réciproque du regard entre une grand-mère et une petite-fille constitue la source d'une quête d'identité de la part de la fille. Un tel reflet valorisé réussit à renforcer une vision d'indépendance féminine et à créer une solidarité psychologique féminine (dans le cas de Schwarz-Bart), tandis que l'échec du reflet brise le miroir ou transforme la filiation en servitude (dans le cas de Rhys). Dans chacun des romans, cependant, cette reproduction du maternage se reflète dans le texte écrit par la fille?.

Il n'est pas sans intérêt d'appliquer les théories féministes de Chodorow, de Gilligan, de Dinnerstein, de Rich et d'Irigaray et l'adaptation de ces théories dans l'étude de Scharfman à l'analyse de quelques œuvres de Gabrielle Roy8. Après avoir montré une conscience féministe dans ses premières nouvelles des années quarante, il semble que Roy soit devenue ensuite une écrivaine plutôt traditionnelle, humaniste, qui excellait dans la création de femmes fictives et qui mettait en relief le mythe de la mère québécoise. Mais cette mère envers qui la narratrice des romans de Roy exprime tant d'orgueil et tant de pitié, cette mère qui souffre seule, reste sans le bénéfice du regard de sa fille: comme dans le roman de Jean Rhys, c'est le cas de Rose-Anna et de Florentine Lacasse du premier roman de Gabrielle Roy, Bonheur d'occasion, paru en 1945.

Puisqu'il n'y avait jamais de rapport chaleureux et ouvert entre RoseAnna et sa mère, Mme Laplante, on peut bien comprendre que celle-là ne veuille pas suivre l'exemple de sa mère. Mais elle ne peut pas faire autrement. Pareillement, sa fille, Florentine, a besoin désespérément, du regard de sa mère, mais héritière de Rose-Anna, elle a peur de l'honnêteté qu'entraîne ce reflet mutuel. La scène révélatrice où les deux femmes se tiennent l'une en face de l'autre - l'une sachant et l'autre soupçonnant la vérité horrifiante de la grossesse de Florentine - témoigne de l'échec de reflet nécessaire entre deux générations de femmes: 
Lorsqu'elle (Florentine) se redressa, pâle, le visage humilié, sa mère la regardait. Elle la regardait comme si elle ne l'avait jamais encore vue, et la découvrait soudain. Elle la regardait avec des yeux agrandis, fixes, et une expression de muette horreur. Sans pitié, sans amitié, sans bonté: rien que de l'horreur plein les yeux. Presque violente, d'une voix qui montait, elle s'écria:

«Mais qu'est-ce que t'as donc, toi! Hier, à matin, pis encore à soir... On dirait que t'es..."

Elle s'était tue et les deux femmes se regardaient comme deux ennemis...

Florentine, la première, abaissa la vue.

Une fois encore elle chercha les yeux de sa mère... la première fois et la dernière fois de sa vie sans doute qu'elle mettait dans son regard cet appel d'être traqué. Mais RoseAnna avait détourné la tête... Elle semblait être devenue une chose inerte, indifférente, à demi enfoncée dans le sommeil.

Alors Florentine, avec une impression de recul infini, se vit toute jeune, gaie, fiévreuse sous le regard de Jean...

Elle tourna sur elle-même... et s'enfuit dans une lame de nuit qui semblait la happer9.

Après des années de silence et d'identification négative (Florentine refuse d'imiter la vie de sa mère), les deux femmes ont besoin de communiquer, surtout au moment de cette crise profonde. On note d'abord l'honnêteté et la vérité terrifiantes qui produisent ce désir de parler. Mais cela finit par créer une barrière de haine, mais aussi d'identification pure, entre les deux femmes. Chacune reste isolée de l'autre mais aussi liée à l'autre dans leur destin de femme. C'est la fille, la première, qui ne peut plus supporter ce regard dont le corps et l'âme ont également besoin. Cette foisci, c'est la mère qui a peur de ces émotions nues. Elle nie le reflet mère-fille et se transforme en objet indifférent. Puisque Florentine ne peut exister qu'aux yeux d'un autre - comme sous le regard de son amant, Jean - et puisqu'elle ne reçoit aucun reflet de la part de sa mère, elle crée son propre reflet, tourne sur elle-même et s'enfuit dans le vent. C'est l'image qui la symbolise à travers tout le roman - un tourbillon qui se mire en lui-même et qui se détruit.

Plus tard, à la veille du mariage de Florentine avec Emmanuel, RoseAnna "guettait un mot, un regard de Florentine...» car elle espère toujours avoir "une explication entre femmes..." Avec "les yeux gonflés d'incertitude», la mère tente de lui parler, mais la fille lui coupe la parole:

Rose-Anna voyait le visage de sa fille dans une petite glace... La bouche était dure, le regard volontaire, presque insolent.

Elle l'examinait avec stupeur. Cette Florentine... lui était une inconnue... 
Elle laissa fuir son regard. L'image de sa vieille mère (Mme Laplante), inflexible et froide, s'imposa à elle, et l'idée lui vint qu'elle lui ressemblait peut-être... (Florentine) faillit se jeter dans les bras de sa mère, mais Rose-Anna, s'étant détournée, hésita une courte minute, puis s'enfuit dans la cuisine10.

Dans un reflet double - le regard dans la glace et le regard du souvenir - de mère à fille pendant trois générations, ces femmes se reconnaissent dans l'image de leur mère en même temps qu'elles refoulent tout lien et toute identité avec elle. Les tentatives de reflet apparaissent toujours, mais tout de suite aussi le rejet et la distance. Il ne reste donc aucun miroir, aucun reflet entre mère et fille:

Florentine ne regardait point en arrière. Elle n'avait eu en partant aucune hésitation, aucun geste ému, pas même un seul regard un peu prolongé. Elle était partie comme si rien ici ne la retenait plus, ne la touchait plus, pensa RoseAnna... si bouleversée qu'elle n'eut plus qu'un instinct: se cacher quelque part, cacher son visage, rester longtemps seule, toute seule 11 .

Les romans de Gabrielle Roy - Bonheur d'occasion, Alexandre Chenevert, la Montagne secrète et la Rivière sans repos - offrent aux lecteurs et aux lectrices une image angoissée de l'être humain, et surtout de la femme-mère. Les protagonistes féminines de la plupart des nouvelles de Roy - celles qui dominent les recueils comme la Petite Poule d'eau, Rue Deschambault, la Route d'Altamont et Ces enfants de ma vie, par exemple - présentent un portrait plus optimiste, quoique superficiel, de la femmemère-institutrice-future écrivaine. Sur cet arrière-plan s'ébauche un reflet mère-fille comparable à celui qu'on trouve dans le roman de Schwarz-Bart, et qui exemplifie le lien entre femmes proposé par les théoristes féministes.

Rue Deschambault, paru en 1957, est un recueil de nouvelles dont la protagoniste principale est une jeune femme, Christine, mise en scène en tant qu'enfant puis adolescente et qui joue le rôle de narratrice adulte. Cette dernière recrée, revit, regarde et juge ses années de jeunesse et surtout la présence inoubliable de sa mère, tandis que la jeune fille elle-même reste la conscience centrale de ce monde renouvelé et interprète tout ce qui se passe autour d'elle. Nous voyons dans ces histoires, donc, des reflets multiples: entre Christine adolescente et Christine la narratrice adulte; entre la jeune fille puis l'adolescente et sa mère; entre Christine femme et le souvenir de sa mère.

Puisque Christine est la dernière enfant qui reste à la maison, il se crée des similitudes entre la jeune fille et sa mère. On note un lien de plus en plus profond et intime dans la plupart de ces nouvelles. Christine et Maman se parlent; elles se regardent; elles cherchent une identité de femme: «Maman prit ma main et la serra comme pour faire passer en moi un mouvement de son âme...12». Christine, jeune ou adulte, emploie sans cesse l'expression, 
«maman et moi», et les mots: «nous», «notre» et «nos». Le rapport entre la mère et la fille - ou le «Je» devient un «Nous» - est ici entièrement conforme aux théories psychologiques féministes.

Il faut mettre en relief deux aspects de ce rapport féminin qui sont développés vers la fin de ce recueil et les rapprocher de la Route d'Altamont, paru en 1966. Ces rapports illustrent l'ouvre des théoriciennes féministes et l'adaptation littéraire qu'en fait Scharfman. Dans «La voix des étangs», après avoir expliqué que c'était Maman qui lui avait légué l'amour de la fiction et du pouvoir merveilleux du mot juste et de la belle phrase, Christine raconte la réaction de sa mère à sá décision de devenir écrivaine:

«Écrire», me dit-elle tristement, «c'est dur... N'est-ce pas se partager en deux, pour ainsi dire: un qui tâche de vivre, l'autre qui regarde, qui juge... Ce don, c'est un peu comme une malchance qui éloigne les autres, qui nous sépare de presque tous...

Écrire... est-ce que ce n'est pas en définitive être loin des autres... être toute seule, pauvre enfant 13 »).

Maman, tout en se mirant dans l'image de sa fille, la comprend bien et se rend compte, instinctivement, de la difficulté d'écrire, surtout pour une femme qui cherche sa propre voix et son propre langage de femme, qui craint surtout cet éloignement des autres et qui reste, donc, souvent silencieuse.

Dans la dernière nouvelle du recueil, "Gagner ma vie», Christine est devenue institutrice - tout en rêvant d'un avenir littéraire - essentiellement pour satisfaire le rêve qu'avait toujours Maman pour elle, rêve qui compensait son propre désir sacrifié: "Quand on se connaît mal encore soimême, pourquoi ne tâcherait-on pas de réaliser le rêve que ceux qui nous aiment font à notre usage! 14 » Après cette réaction typique d'une fille envers sa mère, Christine manifestera un sentiment de culpabilité quand elle ne pourra plus réaliser le rêve maternel. On verra qu'elle tentera de récompenser Maman par une sorte de reproduction de la mère dans ses écrits, dans ce texte particulier et dans le texte de la Route d'Altamont.

Dans Pluie et Vent sur Télumée Miracle de Schwarz-Bart, la grandmère de Télumée, Reine Sans Nom, est à la fois mythique et humaine. Surtout lorsqu'elle raconte à sa petite-fille l'histoire imaginaire et symbolique de la Genèse, elle devient un miroir dans lequel la jeune fille peut chercher son identité de femme créatrice et indépendante15. Pareillement dans la nouvelle, "Ma grand-Mère toute puissante», de la Route d'Altamont, Mémère, vieille grand-mère de la jeune narratrice, Christine, se décide à lui prouver qu'elle est toujours capable, et elle lui crée une poupée («une catin»). L'effet de ce talent créateur sur la jeune fille est profond:

Je grimpai sur ses genoux, je lui jetai mes bras autour du cou, je sanglotai d'un bonheur aigu... Il n'y avait pas de 
limites à ce que savait faire et accomplir cette vieille femme... Je lui criai dans l'oreille:

"Tu es Dieu le Père. Tu es Dieu le Père. Toi aussi, tu sais faire tout de rien...»

Je voyais que malgré tout elle n'était pas offensée de ce que je l'avais comparée à Dieu le Père16.

Il est intéressant de noter que Christine imagine Mémère en tant que Dieu mâle - tout ce qui lui reste d'une éducation religieuse - mais que l'image de Dieu la Mère lui semble plus vraisemblable. Impressionnée par le pouvoir créateur et la grandeur solitaire de cette vieille femme, autrefois distante, la jeune fille la voit, dès lors, comme plus affable. Et dans un reflet renversé, la fière Mémère, se sentant autrefois inutile, commence à se regarder comme moins éloignée.

Dans ce miroir de la grand-mère-Dieu, la petite-fille peut voir, donc, le reflet d'une déesse humaine qui est à la fois sage et protectrice. C'est l'image positive de la femme puisssante et maternelle, qui revalorisera chez Christine l'image de la femme créatrice et restera longtemps dans son esprit:

Dieu le Père, à la grande barbe et à l'air courroucé, céda la place à grand-maman aux yeux fins, rusés et clairvoyants. C'était elle qui, assise dans les nuages, dès lors prenait soin du monde, édictait de sages et justes lois. Or le pauvre monde sur terre s'en trouvait bien.

Longtemps il me resta dans l'idée que ce ne pouvait être un homme sûrement qui eût fait le monde. Mais, peut-être, une vieille femme aux mains extrêmement habiles 17.

Exemple de l'éthique féminine de responsabilité, de soin et de compassion envers la mère, telle que décrite par Gilligan, Maman emmène vivre Mémère à la maison. Très vieille, sans l'activité «divine» d'autrefois et sans plus d'interaction directe avec sa petite-fille, elle perd son identité de grandmère puissante aux yeux de celle-ci. Elle perd même son nom, au point de devenir «une vieille personne»; «la vieille, vieille femme»; «la vieille personne chez nous». Elle retourne à l'état d'altérité d'un objet non-identifié (Dinnerstein) - «ce bloc immobile sous les couvertures...18》.

Mais ce qui confond et intrigue Christine c'est une certaine «confusion à propos des âges», ce qu'elle nomme «un étrange jeu»: elle observe Maman qui prend soin de Mémère comme si la vieille femme était sa fille, mais qui lui pose, en même temps, des questions de fille à sa mère19. Christine essaie aussi de comprendre ce qu'elle appelle «ce va-et-vient d'un être humain à travers le souvenir d'un autre»: en tant qu'interprète à midistance entre ces trois générations de femmes, Maman raconte à la fois la jeunesse et la vieillesse de Mémère à la jeune fille20. Le reflet réciproque de mère à fille entre Mémère et Maman s'effectue; il reste à effectuer le reflet entre grand-mère et petite-fille — sans présence active de la mère - ce qui s'était réalisé au début de cette histoire. 
Cette rencontre commence par le regard de Mémère dont les yeux prouvent enfin son identité de vraie grand-mère et appellent Christine à s'asseoir, à son tour après Maman, près d'elle. Avec une vision vague de sa grand-mère-arbre avec de jeunes arbres nés d'elle, la jeune fille court chercher un album de vieilles photographies et se met à nommer à haute voix les membres de la famille. Ce qu'elle fait instinctivement, donc, c'est de trouver un troisième intermédiaire, aussi "créé» par Mémère, pour effectuer une relation entre deux générations de femmes: la poupée, Maman, et maintenant les photos des descendants de la vieille femme. En plus, surtout après avoir trouvé une photo de Mémère quand elle était jeune et après avoir comparé ces deux "versions» de la même femme, Christine est prête à mieux comprendre ce va-et-vient d'un être humain à travers le temps et à travers l'autre.

$\mathrm{Ce}$ «jeu de la rencontre» ou ce regard en miroir devient plus complexe lorsque Christine aperçoit Maman sur le seuil de la chambre, témoin passif et néanmoins impliqué de cette scène:

Mais pourquoi avait-elle l'air si contente de moi? Je n'avais pourtant fait que jouer, comme elle-même me l'avait enseigné, comme mémère aussi un jour avait joué avec moi... comme nous jouons tous peut-être, les uns avec les autres, a travers la vie, à tâcher de nous rencontrer21.

Le rapport semi-symbiotique entre Mémère et Christine est réalisé plus profondément grâce à la présence de Maman, car les trois générations pourront puiser à la source de puissance de la (grand)mère et de la (petite)fille.

Même après la mort de la plus vieille génération, l'efficacité de ce lien entre femmes durera - ce que raconte la narratrice, Christine, dans la dernière nouvelle de ce recueil, «La route d'Altamont». Tout ce qu'il faut pour faire renaître cette rencontre et ce va-et-vient entre la jeunesse et la vieillesse vieillesse c'est un intermédiaire, cette fois-ci des routes de section sur la plaine canadienne, découvertes par hasard par la fille pour la mère. Ces routes se distinguent par «leur rencontre silencieuse», «leur étonnement à se rencontrer», et n'existent que «pour l'exaltation étrange de l'âme à jouer avec elles quelque jeu puéril et fascinant 22 \%. Une de ces routes mènera la vieille Maman (Eveline) et Christine, maintenant adulte, vers les seules collines du sud du Manitoba (les collines aussi «jouant avec nous un jeu d'attente...23» dans leur rôle d'intermédiaire maternel miroitant) où Maman se souviendra de Mémère et retrouvera en elle-même le bonheur d'un dialogue avec sa propre enfance. Au cours de ce voyage aux sources de la jeunesse, observée par la fille, Maman retournera aussi vers sa mère ou plutôt vers l'image mnémotique de sa mère.

Par suite de la découverte de ces collines, il semble que Maman écoute quelqu'un d'invisible, une voix venue du passé qui l'enlève partiellement à une Christine jalouse de cette triple glace. Mais pour plaire à sa mère, la fille l'emmène une deuxième fois à cet endroit inspirant où dans une description inoubliable du reflet - de la fusion même - entre mère et fille, Eveline s'exprime: 
"C'est avec l'âge mûr que je l'ai rejointe ou qu'elle-même (Mémère) m'a rejointe, comment expliquer cette étrange rencontre hors du temps... Maintenant, peux-tu honnêtement me dire que je ne ressemble pas étonnamment à ce portrait que nous avons de grand-mère à l'âge que j'ai atteint?

Par le caractère aussi, va! (...) Devenue elle, je la comprends. Ah, c'est bien là l'une des expériences les plus surprenantes de la vie. À celle qui nous a donné le jour, on donne naissance à notre tour quand, tôt ou tard, nous l'accueillons enfin dans notre moi. Dès lors, elle habite en nous autant que nous avons habité en elle avant de venir au monde. (...) C'est comme si je lui donnais une voix pour s'exprimer. (...) On se rencontre (...) on finit toujours par se rencontrer, mais si tard 24 !»

Ces belles paroles d'Eveline soulignent admirablement les théories psychologiques féministes de Chodorow, de Dinnerstein, de Rich et de Gilligan. Elles offrent, en plus, une amplification de l'expression d'Irigaray, "se remettre au monde 25 »: ici c'est la mère qui a mis au monde une fille qui restaure le moi, l'âme de cette plus vieille femme lorsque cellelà lui redonne une voix et recommence à se mirer et à s'identifier avec elle. C'est ainsi que la fille se remet aussi au monde.

Mais c'est avant tout la possibilité de redonner une voix à la mère qui relie Eveline à Christine autant qu'à Mémère:

«Car, je (Eveline) te (Christine) regarde et me dit que rien n'est perdu, que tu feras à ma place et mieux que moi ce que j'aurais désiré accomplir...

N'as-tu donc pas encore compris que les parents revivent vraiment en leurs enfants?»

«Je pensais que tu revivais surtout la vie de tes parents à toi.»

«Je revis la leur, je revis aussi avec toi. (...) C'est peut-être la partie de la vie la plus éclairée, située entre ceux qui nous ont précédés et ceux qui nous suivent (...)26.»

Comme dit Carol Gilligan: «Nous nous connaissons en tant qu'êtres humains distincts dans la mesure où nous vivons par rapport aux autres 27 ». Maman revit la vie de sa mère et lui redonne naissance; elle revivra aussi la vie avec sa fille qui la remettra au monde et lui rendra aussi une voix. En effet, Christine (re)créera et sa grand-mère et sa mère, leur redonnera des voix pour s'exprimer par le moyen de ses textes littéraires futurs, déjà annoncés à la fin de la Rue Deschambault et réalisés dans ce texte particulier. Nous arrivons de nouveau à une certaine reproduction du maternage.

Cependant, il faudra, en même temps, que la fille lutte pour accéder à sa propre autonomie de femme - lutte dirigée, par nécessité, contre la mère, devenue martyre, et à cause de l'identification féminine référentielle, 
contre elle-même. De plus en plus au vingtième siècle, les modes de vie indépendants accessibles à la jeune fille continuent à affaiblir le rapport de mère à fille28. L'idée même de cette séparation inévitable entre la mère et la fille - comme dans le cas d'Éveline et de Christine - produira des sentiments de tension.

Eveline admet franchement qu'elle a besoin de sa fille: «Reste jeune et avec moi toujours, ma petite Christine, afin que je ne devienne pas trop vite tout à fait vieille et disputeuse 29 ». Après avoir essayé d'inculquer un sentiment de culpabilité dans le jeune esprit de sa fille, Maman réagit immédiatement à l'annonce que Christine veut séjourner en Europe pour faire une sorte d'apprentissage avant de devenir écrivaine: elle s'éloigne d'abord émotionnellement de sa fille. Ensuite comme dans le reflet réciproque nu entre Rose-Anna et Florentine, Eveline et Christine se mirent avec un regard qui mènera à l'honnêteté, à la cruauté et à l'hostilité: "Devant l'excès de nos propos, nos défenses sont tombées un instant, et nous nous sommes regardées l'une l'autre dans la peine30».

Se sentant coupable de cette séparation anticipée, de cette distance et de cette tension avec sa mère, Christine, une dernière fois avant son départ, l'aide à retrouver Mémère et à se retrouver dans les collines du Manitoba. Mais il semble que ces collines soient plus petites qu'autrefois et qu'elles leur donnent l'impression d'une photographie imparfaite. Il est évident, donc, que le départ anticipé de la fille a dévié ou a brisé le reflet mère-fille. II n'y a plus de ressemblance entre ces trois femmes - l'une avec l'autre ou chacune avec elle-même-comme il y en avait entre les photos de la jeune et de la vieille Mémère et entre les photos de Mémère et d'Eveline. Les intermédiaires (la fille et les collines) ont perdu leur capacité de faire renaître au monde la jeunesse, la mère et le retour à la mère. Tout en se rendant compte de la vérité, Maman ne lance qu'un regard indifférent, défensif aux collines tandis qu'elle accuse Christine d'avoir perdu ses collines «aujourd'hui justement» - lorsqu'elle avait tant besoin de Mémère parce que sa fille se séparait d'elle: «Elle (Eveline) me lança alors un regard si aigu, si désolé, si seul...31», sans reflet.

S'il est vrai que le champ psychologique du moi chez les filles est perméable, malléable, vague et défini par l'attachement à la mère et aux autres, il n'est pas surprenant qu'en ayant sans cesse besoin de ce lien avec sa mère de qui elle s'est séparée, Christine désire se prouver aux yeux de celle-là et la récompenser de la difficulté de l'avoir mise au monde et de l'avoir élevée. Même le moindre manquement à cette responsabilité de fille la fera se sentir coupable:

Pourtant ce n'était rien en regard de ce que je ferais pour elle, si seulement elle m'en donnait le temps. (...) Je me pensais toujours au bord de ce que je voulais devenir à ses yeux avant de lui revenir...

Peut-elle seulement attendre que je sois prête à lui montrer ce dont je voudrais être capable? Et si elle ne le peut pas, ce que je tiens à accomplir aura-t-il seulement encore de la 


\section{valeur à mes yeux 32 ?}

Mais il est trop tard, et Maman est morte de maladie et de chagrin avant le retour de Christine. $\grave{A}$ son tour, la mère avait tellement besoin de se refléter dans le regard de sa fille qu'elle ne pouvait jamais se rétablir de cette séparation et du vide qu'elle en ressentait.

Le sentiment de culpabilité de la fille persistera. Tout ce que Christine fille de sa mère et narratrice peut faire c'est d'accomplir ce dont elle voudrait être capable: (re)créer le reflet-lien mère-fille; (re)créer ou (re)mettre au monde la grand-mère et la mère tout en leur donnant une voix, un langage de femme; (re)jouer la rencontre ultime hors du temps entre ces trois générations de femmes par le moyen de la fiction, de son texte littéraire de fille33.

On peut dire, donc, que ces nouvelles de Gabrielle Roy représentent une quête de la mère, de l'ancêtre maternel et matriarcal. Mais puisque toutes ces routes vont par Altamont, comme l'écrit la narratrice34, de tels voyages serviront aussi à emmener la vieillesse à la jeunesse, Mémère, Eveline et Christine l'une à l'autre, la (grand)mère à la (petite) fille et la (petite) fille à la (grand)mère. De tels textes littéraires serviront à réaliser de nouveau le reflet et la solidarité entre ces générations de femmes pourqu'elles puissent (re)vivre leur rapport interdépendant et semisymbiotique.

1. Nancy Chodorow, The Reproduction of Mothering: Psychoanalysis and the Sociology of Gender, Berkeley, University of California, Press, 1978, pp. 32, 39, 50, 109, 166.

2. Ibid., pp. 93, 110, 127, 166-169, 176.

3. Carol Gilligan, In a Different Voice: Psychological Theory and Women's Development, Cambridge, Harvard University Press, 1982, p. 100. C'est nous qui traduisons. Voir aussi pp. $8,159,160-161$.

4. Dorothy Dinnerstein, The Mermaid and the Minotaur: Sexual Arrangements and Human Malaise, New York, Harper and Row, Publishers, 1976, pp. 106, 107, 110-112, 193.

5. Adrienne Rich, Of Woman Born: Motherhood as Experience and Institution, réimpression, New York, Bantom Books, Inc., 1976, 1981, p. 240. C'est nous qui traduisons.

6. Luce Irigaray, «Quand nos lèvres se parlent», dans Ce sexe qui n'en est pas un, Paris, Les Éditions de Minuit, collection "Critique», 1977, pp. 203-217. Voir aussi Gilligan, p. 127; Dinnerstein, p. 102; Rich, PP. 220-221, 226.

7. Ronnie Scharfman, «Mirroring and Mothering in Simone Schwarz-Bart's Pluie et Vent sur Télumée Miracle et Jean Rhys' Wide Sargasso Sea», Feminist Readings: French Texts/American Contexts, Yale French Studies, ${ }^{\circ}$ 62, 1981, pp. 88-91, 98, 99, 106.

8. Parmi de nombreuses études critiques consacrées à l'oeuvre de Gabrielle Roy, on peut mentionner: Jacques Blais, «L'unité organique de Bonheur d'occasion», Études françaises, vol. VI, $\mathrm{n}^{\circ} 1$, février 1970, pp. 25-50; André Brochu, «Thèmes et structures de Bonheur d'occasion", dans l'Instance critique, Montréal, Leméac, 1974, pp. 206-246; Marc Gagné, Visages de Gabrielle Roy, Montréal, Éditions Beauchemin Limitée, 1973; Albert Le Grand, "Gabrielle Roy ou l'être partagé», Études françaises, $n^{\circ} 2$, juin 1965, pp. 39-65; Gabrielle Pascal, "La condition féminine dans l'ouvre de Gabrielle Roy», Voix et Images, vol. V, ${ }^{\circ}$ 1, automne 1979, pp. 143-163; François Ricard, Gabrielle Roy, Montréal, Éditions Fides, "Ecrivains canadiens d'aujourd'hui», $\mathrm{n}^{\circ} 11$, 1975; Ben Shek, «L'espace et la description symbolique dans les romans 'montréalais' de Gabrielle Roy", Liberté, n ${ }^{\circ} 131,1971, \mathrm{pp}$. 78-96; Paul Socken, “'Le pays de l'amour' in the Works of Gabrielle Roy», Revue de l'Université 
I'Université d'Ottawa, vol. XLVI, n 3, juillet-septembre 1976, pp. 309-323. La revue, Études littéraires, a consacré un numéro spécial à Gabrielle Roy en 1984. En plus, la première introduction critique en anglais consacrée à toute l'oeuvre de Gabrielle Roy sera publiée aux États-Unis en automne 1984: Paula Gilbert Lewis, The Literary Vision of Gabrielle Roy: An Analysis of Her Works, Birmingham, Alabama et Lawrence, Kansas, Summa Publications, Inc.

9. Gabrielle Roy, Bonheur d'occasion, réimpression, Montréal, Librairie Beauchemin Limitée, (c) 1945, 1973, p. 232.

10. Ibid., pp. 307-310.

11. Ibid., p. 314 .

12. Gabrielle Roy, Rue Deschambault, réimpression, Montréal, Librairie Beauchemin Limitée, (C) 1957, 1974, p. 99.

13. Ibid., p. 246.

14. Ibid., p. 284.

15. Scharfman, pp. 92-95; Simone Schwarz-Bart, Pluie et Vent sur Télumée Miracle, Paris, éditions du Seuil, 1972, pp. 76-79.

16. Gabrielle Roy, «Ma grand-Mère toute puissante», la Route d'Altamont, Montréal, Éditions HMH, collection l'Arbre, $\mathrm{n}^{\circ} 10,1966$, pp. 27-28; Paula Gilbert Lewis, "The Resignation of Old Age, Sickness, and Death in the Fiction of Gabrielle Roym, The Americain Review of Canadian Studies, vol. XI, $\mathrm{n}^{\circ} 2$, Autumn 1981, pp. 50-51.

17. Roy, "Ma grand-Mère toute puissante», la Route d'Allamont, p. 31.

18. Ibid., pp. 46, 47, 50, 51; Gilligan. pp. 95, 98, 105, 148; Dinnerstein, pp. 106-107, 110-111. Voir aussi Lewis, p. 51.

19. Roy, “Ma grand-Mère toute puissante», la Route d'Altamont, pp. 51-52.

20. Ibid., p. 37. Voir aussi pp. 36, 49.

21. Gagné, p. 85; Roy, "Ma grand-Mère toute puissante», la Route d'Altamont, p. 57. Voir aussi Scharfman, p. 93; Lewis, p. 52. Ce concept du temps circulaire qui lie les trois générations de femmes se renforce par le souvenir et la comparaison entre Mémère et la petite Rivière Assomption de sa jeunesse au Québec. On peut étudier cette image du temps circulaire chez Roy dans Paula Gilbert Lewis, "The Themes of Memory and Death in Gabrielle Roy's la Route d'Altamont", Modern Fiction Studies, vol. XXII, n 3, Autumn 1976, pp. 457-466.

22. Roy, «La route d'Altamont", la Route d'Altamont, pp. 197-198.

23. Ibid., p. 203.

24. Ibid., pp. 226-227. Voir aussi Gabrielle Roy, De quoi t'ennuies- $t u$, Eveline?, Montréal Éditions du Sentier, 1982, p. 34; Lewis, "The Themes of Memory and Death», pp. 458, 462-464; Lewis, "The Resignation of Old Age», pp. 51, 61, 62; Paula Gilbert Lewis, «Street of Riches and The Road Past Altamont: The Feminine World of Gabrielle Roy", Journal of Women's Studies in Literature, vol. I, $\mathrm{n}^{\circ} 2$, Spring 1979, p. 138.

25. Cette expression se trouve dans Luce Irigaray, Et l'une ne bouge pas sans l'autre, Paris, Éditions de Minuit, 1979. Elle est traduite et expliquée en anglais par Hélène Vivienne Wenzel, "And the One Doesn't Stir without the Other», par Luce Irigaray, Signs: Journal of Women in Culture and Society, vol. VIII, $\mathrm{n}^{\circ} 1$, Autumn 1981, p. 65. Voir aussi Chodorow, pp. 39, 93; Dinnerstein, pp. 106, 107, 110-112; Gilligan, pp. 149, 173.

26. Roy, "La route d'Altamont», la Route d'Altamont, p. 236. Voir aussi Lewis, «The Themes of Memory and Death», p. 466.

27. Gilligan, p. 63. C'est nous qui traduisons.

28. Voir: Dinnerstein, p. 193; Gilligan, p. 51; Rich, pp. 236-237, 238. Dans son étude sur les possibilités offertes à la femme au $\mathrm{XX}^{\mathrm{e}}$ siècle, Rich cite Carroll Smith-Rosenberg, "The Female World of Love and Ritual; Relations between Women in Nineteenth-Century America», Signs, vol. $1, \mathrm{n}^{\circ} 1,1975$, pp. 1-29.

29. Roy, "La route d'Altamont », la Route d'Altamont, p. 229.

30. Ibid., p. 239. 
31. Ibid., pp. 251-252.

32. Ibid., pp. 254-255, 248-249. Voir aussi Chodorow, pp. 93, 126, 135, 167-168; Dinnerstein, pp. 106, 107, 110-111; Gilligan, pp. 95, 98, 105, 148, 159, 173; Lewis, «The Feminine World", pp. 138-139.

33. À propos de Gabrielle Roy, François Ricard dit que l'écrivain(e) qui «part pour écrire» rencontre la solitude et la séparation, mais que cet(te) écrivain(e) «revient en écrivant». Ce retour indique une tentative de réconciliation (Voir Ricard, p. 29). On peut décrire, donc, cette réconciliation en termes de (re)création de la (grand) mère par la fille.

34. Roy, «La route d'Altamont», la Route d'Altamont, p. 255. 